\title{
Statistical Inference of Sine Inverse Rayleigh Distribution
}

\author{
Abdullah Ali H. Ahmadini* \\ Department of Mathematics, Faculty of Science, Jazan University, Jazan, Saudi Arabia \\ *Corresponding Author: Abdullah Ali H. Ahmadini. Email: aahmadini@jazanu.edu.sa \\ Received: 07 May 2021; Accepted: 08 June 2021
}

\begin{abstract}
We study in this manuscript a new one-parameter model called sine inverse Rayleigh (SIR) model that is a new extension of the classical inverse Rayleigh model. The sine inverse Rayleigh model is aiming to provide more fitting for real data sets of purposes. The proposed extension is more flexible than the original inverse Rayleigh (IR) model and it hasmany applications in physics and medicine. The sine inverse Rayleigh distribution can havea uni-model and right skewed probability density function (PDF). The hazard rate function (HRF) of sine inverse Rayleigh distribution can be increasing and J-shaped. Several of thenew model's fundamental characteristics, namely quantile function, moments, incompletemoments, Lorenz and Bonferroni Curves are studied. Four classical estimation methods forthe population parameters, namely least squares (LS), weighted least squares (WLS), maximum likelihood (ML), and percentile (PC) methods are discussed, and the performanceof the four estimators (namely LS, WLS, ML and PC estimators) are also compared bynumerical implementations. Finally, three sets of real data are utilized to compare the behavior of the four employed methods for finding an optimal estimation of the new distribution.
\end{abstract}

Keywords: Sine generated family; inverse Rayleigh distribution; classic estimation methods; applications

\section{Introduction}

Reference [1] investigated a significant distribution in analysis of lifetime, namely the inverse Rayleigh (IR) model. The IR model's considered probability density function (PDF) and the corresponding distribution function (CDF) are given by

$g(y)=2 \theta y^{-3} e^{-\frac{\theta}{y^{2}}}, \quad \theta>0, \quad y>0$.

and

$G(y)=e^{-\frac{\theta}{y^{2}}}, \quad \theta>0, y>0$.

Much effort has been invested in the literature on estimating the IR model; see, for instance, [2-10].

In last years, several extensions for the IR model were established by means of various generalization methods such as beta IR, transmuted IR (TIR), modified IR, transmuted modified IR,

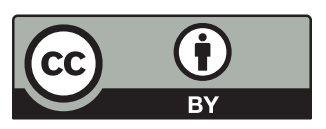

This work is licensed under a Creative Commons Attribution 4.0 International License, which permits unrestricted use, distribution, and reproduction in any medium, provided the original work is properly cited. 
Kumaraswamy exponentiated IR, weighted IR and odd Fréchet IR and half logistic IR models as mentioned in [2-9].

In the last years, many different statisticians are attracted by generated families of distributions as: sine generated (S-G) by [10], Type II half logistic-G by [11], odd Frèchet-G by [12], truncated Cauchy power-G by [13], transmuted odd Fréchet-G by [14], exponentiated M-G by [15], Topp-Leone odd Fréchet-G studied in [16], among others.

For instance, for S-G the CDF and the PDF are

$F(y ; \xi)=\sin \left[\frac{\pi}{2} G(y ; \xi)\right], \quad x \in R$.

and

$f(y ; \xi)=\frac{\pi}{2} g(y ; \xi) \cos \left[\frac{\pi}{2} G(y ; \xi)\right], \quad x \in R$.

where $g(y ; \xi)$ considers a PDF of baseline distribution.

Now, we put forward a novel lifetime model with one parameter named sine inverse Rayleigh (SIR) distribution, whose CDF with parameter $\theta$ is obtained by employing (2) in (3) as

$F(y)=\sin \left[\frac{\pi}{2} e^{-\frac{\theta}{y^{2}}}\right], \quad y>0, \quad \theta>0$,

Likewise, by combining (1), (2) and (4), one obtains the corresponding PDF to (5) as

$f(y)=\frac{\pi \theta}{2 y^{3}} e^{-\frac{\theta}{y^{2}}} \cos \left[\frac{\pi}{2} e^{-\frac{\theta}{y^{2}}}\right], \quad y>0, \quad \theta>0$,

where $\theta$ is a scale parameter.

When the random variable Y has an SIR model, one can define X's hazard rate function (HRF), reversed HRF, cumulative HRF, and survival function (SF) as

$$
\begin{aligned}
& R(y)=1-\sin \left[\frac{\pi}{2} e^{-\frac{\theta}{y^{2}}}\right], \\
& h(y)=\frac{\frac{\pi \theta}{y^{3}} e^{-\frac{\theta}{y^{2}}} \cos \left[\frac{\pi}{2} e^{-\frac{\theta}{y^{2}}}\right]}{1-\sin \left[\frac{\pi}{2} e^{-\frac{\theta}{y^{2}}}\right]}, \\
& \tau(y)=\frac{\pi \theta}{y^{3}} e^{-\frac{\theta}{y^{2}}} \cot \left[\frac{\pi}{2} e^{-\frac{\theta}{y^{2}}}\right],
\end{aligned}
$$

and

$H(y)=-\ln \left(1-\sin \left[\frac{\pi}{2} e^{-\frac{\theta}{y^{2}}}\right]\right)$

Figs. 1 and 2 present the PDF and HRF plots of the SIR distribution, respectively, for various $\theta$ values.

Figs. 1 and 2 exhibit that the SIR distribution can have a uni-model and right skewed PDF, while its HRF can be J-shaped and increasing. 


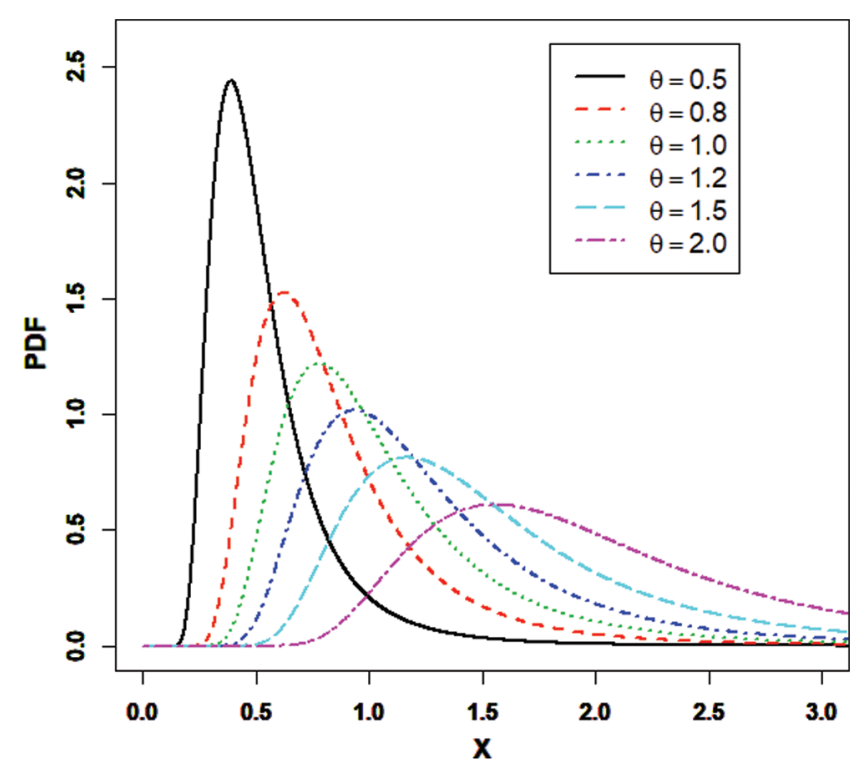

Figure 1: Plots of the PDF the SIR distribution

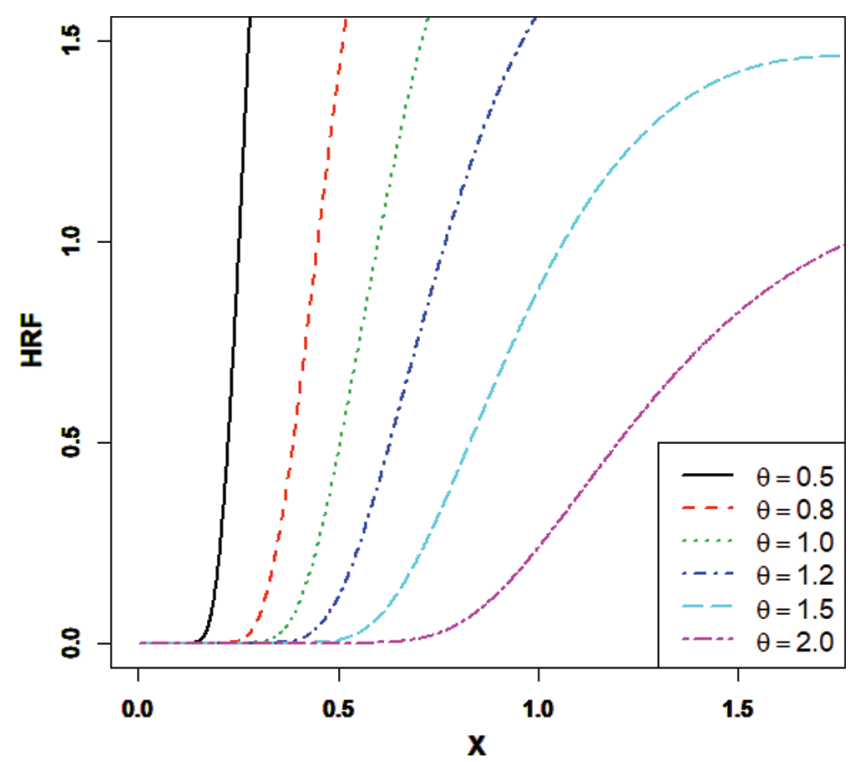

Figure 2: Plots of the HRF the SIR distribution

The remaining parts of this manuscript are presented as follows. Section 2 introduces structural characteristics of SIR distribution including; quantile function, moments, incomplete moments, and Lorenz and Bonferroni curves. Section 3 discusses some estimators for SIR distribution parameters on the basis of four different methods of estimations of least squares (LS), weighted least squares (WLS), maximum likelihood (ML), and percentile (PC). Simulation schemes are performed in Section 4. Three sets of data for real-life applicationare utilized for comparing the behavior of the four methods of estimating the new distribution in Section 5. Several concluding remarks close the work. 


\section{Fundamental Properties}

We study in this part several statistical characteristics of the SIR model.

\subsection{Quantile Function}

If $\mathrm{Y} \sim \mathrm{SIR}$ then, the quantile function of SIR is

$Q(u)=\sqrt{\theta\left[\ln \left(\frac{\pi}{2 \operatorname{Arcsin}(u)}\right)\right]^{-1}}$.

and by taking $\mathrm{u}=0.5$ we get the median $(\mathrm{M})$ as $M=\sqrt{\theta[\ln (3)]^{-1}}$.

\subsection{Moments}

Theorem 1: Assume that $\mathrm{Y}$ is an r.v. from SIR, thus the $r^{\text {th }}$ moment of SIR distribution is

$\dot{\mu}_{r}=\sum_{i=0}^{\infty} \frac{\theta^{\frac{r}{2}-1} \mathrm{C} \Gamma\left(1-\frac{r}{2}\right)}{2(2 i+1)^{1-\frac{r}{2}}}, \quad \frac{r}{2}<1$.

Proof: Assume that $\mathrm{Y}$ is an r. v. with pdf (6). One can determine $r^{\text {th }}$ moments of SIR distribution from

$\dot{\mu}_{r}=\int_{0}^{\infty} y^{r} f(y) d y=\frac{\pi \theta}{2} \int_{0}^{\infty} y^{r-3} e^{-\frac{\theta}{y^{2}}} \cos \left[\frac{\pi}{2} e^{-\frac{\theta}{y^{2}}}\right] d y$,

By inserting the expansion $\cos [G(y)]=\sum_{i=0}^{\infty} \frac{(-1)^{i}}{(2 i) !}[G(y)]^{2 i}, n$ to the previous equation then,

$\dot{\mu}_{r}=\sum_{i=0}^{\infty} \frac{\theta(-1)^{i}}{(2 i) !}\left(\frac{\pi}{2}\right)^{2 i+1} \int_{0}^{\infty} y^{r-3} e^{-(2 i+1)\left(\frac{\theta}{y^{2}}\right)} d y$,

The last equation can be rewritten as

$\dot{\mu}_{r}=\sum_{i=0}^{\infty} \mathrm{C} \int_{0}^{\infty} y^{r-3} e^{-(2 i+1)\left(\frac{\theta}{y^{2}}\right)} d y$.

where $\mathrm{C}=\sum_{i=0}^{\infty} \frac{\theta(-1)^{i}}{(2 i) !}\left(\frac{\pi}{2}\right)^{2 i+1}$.

Let $Z=\frac{\theta}{y^{2}}$, then

$\dot{\mu}_{r}=\sum_{i=0}^{\infty} \theta^{\frac{r}{2}-1} \mathrm{C} \int_{0}^{\infty} z^{-\frac{r}{2}} e^{-(2 i+1) \mathrm{z}} d z$

Then,

$\dot{\mu}_{r}=\sum_{i=0}^{\infty} \frac{\theta^{\frac{r}{2}-1} \mathrm{C} \Gamma\left(1-\frac{r}{2}\right)}{2(2 i+1)^{1-\frac{r}{2}}}$. 
The mgf of $\mathrm{Y}$ is

$M_{X}(t)=\sum_{r=0}^{\infty} \frac{t^{r}}{r !} \mu_{r}=\sum_{r, i=0}^{\infty} \frac{t^{r} \theta^{\frac{r}{2}-1} \mathrm{C} \Gamma\left(1-\frac{r}{2}\right)}{2 r !(2 i+1)^{1-\frac{r}{2}}}$.

obtain the incomplete moments, denoted by $\varphi_{s}(t)$, of the SIR distribution as follows, where $\varphi_{s}(t)$, defined by $\varphi_{s}(t)=\int_{0}^{t} f(y ; \varphi) d y$.

Using (8), $\varphi_{s}(t)$ will be as given

$\varphi_{s}(t)=\sum_{i=0}^{\infty} C \int_{0}^{t} y^{s-3} e^{-(2 i+1)\left(\frac{\theta}{y^{2}}\right)} d y=\sum_{i=0}^{\infty} \frac{\theta^{\frac{s}{2}-1} \mathrm{C} \Gamma\left(1-\frac{s}{2},(2 i+1)\left(\frac{\theta}{t^{2}}\right)\right)}{2(2 i+1)^{1-\frac{s}{2}}}$,

where $\Gamma(s, t)=\int_{0}^{t} y^{s-1} \mathrm{e}^{-y} d y$ denotes the lower incomplete gamma function.

The Lorenz curve and the Bonferroni curve are generated, respectively, from following equations

$L_{F}(x)=\frac{\varphi_{1}(y)}{E(Y)}=\frac{\sum_{i=0}^{\infty} \frac{\theta^{-\frac{1}{2}} \mathrm{C} \Gamma\left(\frac{1}{2},(2 i+1)\left(\frac{\theta}{t^{2}}\right)\right)}{2(2 i+1)^{\frac{1}{2}}}}{\sum_{i=0}^{\infty} \frac{\theta^{-\frac{1}{2}} \mathrm{C} \Gamma\left(\frac{1}{2}\right)}{2(2 i+1)^{\frac{1}{2}}}}=\frac{\sum_{i=0}^{\infty} \frac{\mathrm{C} \Gamma\left(\frac{1}{2},(2 i+1)\left(\frac{\theta}{t^{2}}\right)\right)}{(2 i+1)^{\frac{1}{2}}}}{\sum_{i=0}^{\infty} \frac{\mathrm{C} \sqrt{\pi}}{(2 i+1)^{\frac{1}{2}}}}$,

and

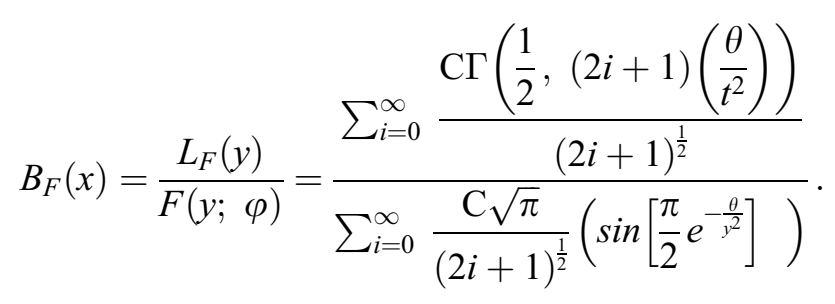

The Zenga curves are given by

$A_{F}(y)=1-\frac{\mu^{-}(y)}{\mu^{+}(y)}$

where

$\mu^{-}(x)=L_{F}(y)$

and

$\mu^{+}(x)=\frac{\int_{t}^{\infty} f(y ; \varphi) d y}{1-F(y)}$. 


\section{Statistical Inference}

The population parameters involved in the SIR model can be estimated by using four different methods of estimation namely; ML, LS, WLS and PC methods.

\subsection{Estimators}

To obtain the MLEs of the SIR model with a parameter $\theta$, let $Y_{1}, \ldots, Y_{n}$ be observed values of this model. The log-likelihood function denoted by $\ell$, can be expressed as

$\ell=n \log \frac{\pi}{2}+n \log \theta-3 \sum_{i=1}^{n} \log \left(y_{i}\right)-\theta \sum_{i=1}^{n}\left(y_{i}\right)^{-2}+\sum_{i=1}^{n} \log \left(\cos \left[\frac{\pi}{2} e^{-\theta\left(y_{i}\right)^{-2}}\right]\right)$.

The ML equation of the SIR model then becomes

$\frac{\partial \ell}{\partial \theta}=\frac{n}{\theta}-\sum_{i=1}^{n}\left(y_{i}\right)^{-2}+\frac{\pi}{2} \sum_{i=1}^{n}\left(y_{i}\right)^{-2} e^{-\theta\left(y_{i}\right)^{-2}} \tan \left[\frac{\pi}{2} e^{-\theta\left(y_{i}\right)^{-2}}\right]$.

The MLEs of $\theta$ are then obtained by equating $\partial \ell / \partial \theta$ with zero and solving simultaneously these equations.

\subsection{LS and WLS Estimators}

Let $Y_{1}, Y_{2}, \ldots, Y_{n}$ be an n-sized random sample (RS) from SIR model and denote the ordered samples in the RS by $Y_{(1)}, Y_{(2)}, \ldots, Y_{(n)}$. The expectation and variance of this model do not depend the unknown parameter given by

$E\left(F\left(Y_{(i)}\right)\right)=\frac{i}{n+1}$, and $\operatorname{var}\left(F\left(Y_{(i)}\right)\right)=\frac{i(n-i+1)}{(n+1)^{2}(n+2)}$,

In the above equations, $F\left(Y_{(i)}\right)$ represents the CDF of the model while $Y_{(i)}$ represents the $i^{\text {th }}$ order statistic (OS). Thus, the LSEs can be determined by obtaining the least sum of squared errors as follows,

$\sum_{i=1}^{n}\left[F_{(i)}(y)-\frac{i}{n+1}\right]^{2}$

regarding $\theta$. Thus, the LSEs of the population parameter $\theta$ of SIR follow by calculating the minimum of the sum

$\sum_{i=1}^{n}\left[\sin \left[\frac{\pi}{2} e^{-\frac{\theta}{\left(y_{(i)}\right)^{2}}}\right]-\frac{i}{n+1}\right]^{2}$

regarding $\theta$. Moreover, the LSEs of $\theta$ can be determined from

$\sum_{i=1}^{n}\left[\sin \left[\frac{\pi}{2} e^{-\frac{\theta}{\left(y_{(i)}\right)^{2}}}\right]-\frac{i}{n+1}\right]\left(\frac{1}{\left(y_{(i)}\right)^{2}} e^{-\frac{\theta}{\left(y_{(i)}\right)^{2}}} \cos \left[\frac{\pi}{2} e^{-\frac{\theta}{\left(y_{(i)}\right)^{2}}}\right]\right)=0$.

The WLSEs of $\theta$ can then be derived by calculating the minimum of the sum

$\sum_{i=1}^{n} \frac{(n+1)^{2}(n+2)}{i(n-i+1)}\left[\sin \left[\frac{\pi}{2} e^{-\frac{\theta}{\left(y_{(i)}\right)^{2}}}\right]-\frac{i}{n+1}\right]^{2}$, 
regarding $\theta$. Moreover, one can determine the WLSEs of $\theta$ from

$$
\sum_{i=1}^{n} \frac{(n+1)^{2}(n+2)}{i(n-i+1)}\left[\sin \left[\frac{\pi}{2} e^{-\frac{\theta}{\left(y_{(i)}\right)^{2}}}\right]-\frac{i}{n+1}\right]\left(\frac{1}{\left(y_{(i)}\right)^{2}} e^{-\frac{\theta}{\left(y_{(i)}\right)^{2}}} \cos \left[\frac{\pi}{2} e^{-\frac{\theta}{\left(y_{(i)}\right)^{2}}}\right]\right)=0 .
$$

\subsection{PC Estimator (PCEs)}

Let $Y_{1}, \ldots, Y_{n}$ denote an RS taken from SIR and assume that $Y_{(1)}<Y_{(2)}<\ldots<Y_{(n)}$ is the corresponding OS. The PCEs of parameter $\theta$ are calculated by minimizing the next

$\sum_{i=1}^{n}\left[\ln \left(\frac{i}{n+1}\right)-\ln \left(\sin \left[\frac{\pi}{2} e^{-\frac{\theta}{\left(y_{(i)}\right)^{2}}}\right]\right)\right]^{2}$,

regarding $\theta$.

\section{Numerical Results}

We generate $3000 \mathrm{RS} Y_{1}, \ldots, Y_{n}$ of sizes $n=10,20,30$ and 50 from SIR were generated. Three different values of the parameter $\theta$ are chosen:

The parameter $\theta$ 's ML, LS, WLS and PC estimates are calculated. Subsequently, the MSEs of the estimate of the unknown parameter are determined. Numerical results are mentioned in Tabs. 1-3 and the following observations can be made.

- The MSEs of ML estimates of $\theta$ are the lowest among all determined MSEs in almost every case.

- The MSEs of all the estimates decrease with increasing sample sizes.

Table 1: Statistical inference of SIR model for ML, LS, WLS and PC estimates when $\theta=0.5$

\begin{tabular}{|c|c|c|c|c|c|c|c|c|}
\hline \multirow[t]{2}{*}{$n$} & \multicolumn{2}{|c|}{ MLEs } & \multicolumn{2}{|c|}{ LSEs } & \multicolumn{2}{|c|}{ WLSEs } & \multicolumn{2}{|c|}{ PCEs } \\
\hline & Estimates & MSEs & Estimates & MSEs & Estimates & MSEs & Estimates & MSEs \\
\hline 10 & 0.527 & 0.018 & 0.521 & 0.023 & 0.519 & 0.022 & 0.458 & 0.020 \\
\hline 20 & 0.513 & 0.008 & 0.509 & 0.009 & 0.511 & 0.009 & 0.473 & 0.010 \\
\hline 30 & 0.509 & 0.005 & 0.506 & 0.006 & 0.503 & 0.006 & 0.475 & 0.007 \\
\hline 50 & 0.506 & 0.003 & 0.505 & 0.004 & 0.504 & 0.0032 & 0.477 & 0.004 \\
\hline
\end{tabular}

Table 2: Statistical inference of SIR model for ML, LS, WLS and PC estimates when $\theta=0.8$

\begin{tabular}{|c|c|c|c|c|c|c|c|c|}
\hline \multirow[t]{2}{*}{$n$} & \multicolumn{2}{|c|}{ MLEs } & \multicolumn{2}{|c|}{ LSEs } & \multicolumn{2}{|c|}{ WLSEs } & \multicolumn{2}{|c|}{ PCEs } \\
\hline & Estimates & MSEs & Estimates & MSEs & Estimates & MSEs & Estimates & MSEs \\
\hline 10 & 0.841 & 0.047 & 0.836 & 0.061 & 0.834 & 0.057 & 0.742 & 0.052 \\
\hline 20 & 0.821 & 0.021 & 0.821 & 0.027 & 0.810 & 0.0213 & 0.745 & 0.026 \\
\hline 30 & 0.814 & 0.013 & 0.811 & 0.015 & 0.808 & 0.014 & 0.751 & 0.017 \\
\hline 50 & 0.807 & 0.008 & 0.805 & 0.009 & 0.807 & 0.0084 & 0.763 & 0.010 \\
\hline
\end{tabular}


Table 3: Statistical inference of SIR model for ML, LS, WLS and PC estimates when $\theta=1.5$

\begin{tabular}{|c|c|c|c|c|c|c|c|c|}
\hline \multirow[t]{2}{*}{$n$} & \multicolumn{2}{|c|}{ MLEs } & \multicolumn{2}{|c|}{ LSEs } & \multicolumn{2}{|c|}{ WLSEs } & \multicolumn{2}{|c|}{ PCEs } \\
\hline & Estimates & MSEs & Estimates & MSEs & Estimates & MSEs & Estimates & MSEs \\
\hline 10 & 1.585 & 0.169 & 1.555 & 0.201 & 1.553 & 0.182 & 1.386 & 0.177 \\
\hline 20 & 1.543 & 0.077 & 1.533 & 0.080 & 1.521 & 0.078 & 1.404 & 0.083 \\
\hline 30 & 1.525 & 0.048 & 1.522 & 0.061 & 1.519 & 0.052 & 1.413 & 0.064 \\
\hline 50 & 1.516 & 0.027 & 1.512 & 0.034 & 1.510 & 0.030 & 1.434 & 0.037 \\
\hline
\end{tabular}

\section{Applications to Real Data}

Further we employ three real data sets for assessing the goodness-of-fit of the SIR model with the purpose of comparing the performance of the considered estimation methods.

We consider criteria including maximum likelihood (denoted by $-\hat{\ell}$,) $(\mathrm{B} 1)$, the Akaike information criterion (AIC) (B2), the consistent AIC (B3), the Schwarz criterion (B4) and the Hannan-Quinn information criterion (B5). The model that has lowest B1-B5 values is deemed the best one with respect to fitting the real data.

The first data set: contains the survival time (days) of 72 guinea pigs with virulent tubercle bacilli infection, originally presented in [17].

The second data set: consists the waiting time (minutes) of 100 bank customers before they were served, as presented in [18].

The third data set: contains 100 results on the breaking stress (Gba) of carbon fibers, as presented in [19].

The parameters of SIR are estimated by the four estimation methods, namely the ML, LS, WLS and PC estimation methods. The efficiency of the estimation methods is the same in the three data sets as shown in Tabs. 4-6. We see that among the estimation methods adopted, ML provides the best results for the included data sets.

Table 4: The goodness of fit statistics for parameters estimated under various methods for the first data set

\begin{tabular}{lllllll}
\hline Method & $\hat{\theta}$ & B1 & B2 & B3 & B4 & B5 \\
\hline MLE & $\mathbf{0 . 7 8 3}$ & $\mathbf{2 6 4 . 0 4 6}$ & $\mathbf{5 3 0 . 0 9 2}$ & $\mathbf{5 2 9 . 9 5}$ & $\mathbf{5 6 2 . 9 6 8}$ & $\mathbf{5 3 0 . 1 4 9}$ \\
LSE & 2.377 & 388.36 & 778.72 & 780.435 & 782.533 & 778.894 \\
WLSE & 2.3774 & 388.394 & 778.789 & 780.503 & 782.601 & 778.963 \\
PCE & 0.051 & 517.874 & 1038 & 1039 & 1042 & 1038 \\
\hline
\end{tabular}

Table 5: The goodness of fit statistics for parameters estimated under various methods for the second data set

\begin{tabular}{lllllll}
\hline Method & $\hat{\theta}$ & B1 & B2 & B3 & B4 & B5 \\
\hline MLE & $\mathbf{2 1 . 5 1}$ & $\mathbf{5 2 3 . 4 9 1}$ & $\mathbf{1 0 4 9}$ & $\mathbf{6 8 4 . 8 0 6}$ & $\mathbf{6 8 6 . 9 1 5}$ & $\mathbf{1 0 5 0}$ \\
LSE & 66.133 & 693.104 & 1388 & 1390 & 1392 & 1388 \\
WLSE & 66.097 & 692.908 & 1388 & 1390 & 1392 & 1388 \\
PCE & 4.117 & 684.116 & 1370 & 1051 & 1053 & 1370 \\
\hline
\end{tabular}


Table 6: The goodness of fit statistics for parameters estimated under various methods for the third data set

\begin{tabular}{lllllll}
\hline Method & $\hat{\theta}$ & B1 & B2 & B3 & B4 & B5 \\
\hline MLE & $\mathbf{4 . 8 0 6}$ & $\mathbf{2 4 6 . 3 6 7}$ & $\mathbf{4 9 4 . 7 3 4}$ & $\mathbf{4 9 6 . 7 3 4}$ & $\mathbf{4 9 5 . 7 8 9}$ & $\mathbf{4 9 4 . 7 7 5}$ \\
LSE & 6.799 & 258.26 & 518.52 & 520.52 & 522.628 & 518.643 \\
WLSE & 6.4747 & 255.018 & 512.036 & 512.036 & 513.09 & 512.077 \\
PCE & 1.368 & 348.028 & 698.056 & 698.056 & 699.11 & 698.097 \\
\hline
\end{tabular}

\section{Conclusion}

We study a new model called SIR. Some fundamental characteristics of the model are investigated. We estimate the model parameters according to the ML, LS, WLS and PC methods. Numerical experiments are carried out in order to comparatively explore the performance of the four different estimation methods. Numerical results show that ML performs better than LS, WLS and PC in almost all considered situations. Applications on three sets of real data indicate that the the ML method is superior in terms of the fits to the LS, WLS and PC methods.

Acknowledgement: The author is grateful to the academic editor and all four reviewers for their valuable comments that have contributed to significantly improve the quality on the article. I would like to thank TopEdit (www.topeditsci.com) for English language editing of this manuscript.

Funding Statement: The author received no specific funding for this study.

Conflicts of Interest: The author declare no conflict of interest.

\section{References}

[1] V. N. Trayer, in Proceedings of the Academy of Science Belarus, Doklady Acad, Nau, Belorus, U.S.S.R., 1964.

[2] J. Leao, H. Saulo, M. Bourguignon, J. Cintra, L. Rego et al., "On some properties of the beta inverse Rayleigh distribution," Chilean Journal of Statistics, vol. 4, pp. 111-131, 2013.

[3] A. Ahmad, S. P. Ahmad and A. Ahmed "Transmuted inverse Rayleigh distribution: A generalization of the inverse Rayleigh distribution," Mathematical Theory and Modeling, vol. 4, pp. 90-98, 2014.

[4] M. S. Khan, "Modified inverse Rayleigh distribution," International Journal of Computer Applications, vol. 87, pp. 28-33, 2014.

[5] M. S. Khan and R. King, "Transmuted modified inverse Rayleigh distribution," Austrian Journal of Statistics, vol. 44, pp. 17-29, 2015.

[6] M. A. Haq "Kumaraswamy exponentiated inverse Rayleigh distribution," Mathematical Theory and Modeling, vol. 6, pp. 93-104, 2016.

[7] K. Fatima and S. P. Ahmad, "Weighted inverse Rayleigh distribution," International Journal of Statistics and Systems, vol. 12, pp. 119-137, 2017.

[8] M. Elgarhy and S. Alrajhi, "The odd fréchet inverse Rayleigh distribution: Statistical properties and applications," Journal of Nonlinear Sciences \& Applications, vol. 12, pp. 291-299, 2018.

[9] A. M. Almarashi, M. M. Badr, M. Elgarhy, F. Jamal and Ch. Chesneau, "Statistical inference of the half-logistic inverse Rayleigh distribution," Entropy, vol. 22, pp. 1-23, 2020.

[10] D. Kumar, U. Singh and S. K. Singh, "A New distribution using sine function-its application to bladder cancer patients data," Journal of Statistics Applications \& Probability, vol. 4, no. 3, pp. 417-427, 2015. 
[11] A. S. Hassan, M. Elgarhy and M. Shakil, "Type II half logistic family of distributions with applications," Pakistan Journal of Statistics and Operation Research, vol. 13, no. 2, pp. 245-264, 2017.

[12] M. A. Haq and M. Elgarhy, "The odd frèchet-g family of probability distributions," Journal of Statistics Applications and Probability, vol. 7, pp. 185-201, 2018.

[13] M. A. Aldahlan, F. Jamal, C. Chesneau, M. Elgarhy and I. Elbatal, "The truncated Cauchy power family of distributions with inference and applications," Entropy, vol. 22, pp. 1-25, 2020.

[14] M. M. Badr, I. Elbatal, F. Jamal, C. Chesneau and M. Elgarhy, "The transmuted odd fréchet-g family of distributions: Theory and applications," Mathematics, vol. 8, pp. 1-20, 2020.

[15] R. A. Bantan, C. Chesneau, F. Jamal and M. Elgarhy, "On the analysis of new COVID-19 cases in Pakistan using an exponentiated version of the M family of distributions," Mathematics, vol. 8, pp. 1-20, 2020.

[16] S. AlMarzouki, F. Jamal, C. Chesneau and M. Elgarhy, "Topp-leone odd fréchet generated family of distributions with applications to COVID-19 data sets," Computer Modeling in Engineering \& Sciences, vol. 125, no. 1, no. 8, pp. 437-458, 2020.

[17] T. Bjerkedal, "Acquisition of resistance in Guinea pigs infected with different doses of virulent tubercle bacilli," American Journal of Epidemiology, vol. 72, pp. 130-148, 1960.

[18] M. E. Ghitany, B. Atieh and S. Nadarajah, "Lindley distribution and its applications," Mathematics and Computers in Simulation, vol. 78, pp. 493-506, 2008.

[19] M. D. Nichols and W. J. Padgett, "A bootstrap control chart for weibull percentiles," Quality and Reliability Engineering International, vol. 22, pp. 141-151, 2006. 